\title{
HIGHLIGHT
}

\section{Discovery of a lipodystrophy gene: one answer, one hundred questions}

\author{
Anath Shalev \\ Diabetes Branch, NIDDK, National Institutes of Health, Bethesda, Maryland 20892, USA; Email: anaths@intra.niddk.nih.gov
}

Lipodystrophy is a rare and heterogenous disorder characterized by absence or loss of adipose tissue from different parts of the body. Classification of the different lipodystrophy syndromes is based mainly on whether the disease is congenital/familial or acquired, and whether the fat loss is generalized or partial. Since the first patient with lipodystrophy was described 115 years ago (1) much has been learned about the clinical features of this condition. However, until recently, little was known about the etiology and pathogenesis of these syndromes. Two groups have now identified the gene mutations responsible for familial partial lipodystrophy. Surprisingly, all the mutations were found in lamin $A / C$, a gene on human chromosome 1q21-22 encoding a nuclear envelope protein $(2,3)$. The gene for congenital generalized lipodystrophy was mapped to human chromosome 9q34 (4).

While these findings provide some insight into the genetic causes of familial lipodystrophy, they also raise many new questions. In order to better understand these recent discoveries and their implications, it is helpful to review briefly what is known about lipodystrophies (5) and lamins (6-9) and about their potential relationship. Lipodystrophies are divided into familial and acquired categories. Familial lipodystrophies can further be subdivided into two major groups: congenital generalized lipodystrophy (Berardinelli-Seip syndrome) and familial partial lipodystrophy (Dunnigan variety) (Table 1).

Congenital generalized lipodystrophy (CGL) is an autosomal recessive disorder characterized by almost complete absence of adipose tissue from birth. All metabolically active fat (subcutaneous, intra-abdominal, intrathoracic, bone marrow, parathyroid glands) is missing whereas mechanical fat (palms, soles, orbits, buccal region, tongue) is preserved.

Familial partial lipodystrophy (FPLD) has an autosomal dominant inheritance, but the onset of symptoms occurs in puberty when patients start losing subcutaneous fat from the extremities, and typically accumulate fat in the neck and face. The rest of the metabolically active adipose tissue remains normal.

The two main syndromes of acquired lipodystrophy are acquired generalized lipodystrophy (Lawrence syndrome) and acquired partial lipodystrophy (BarraquerSimons syndrome).

Acquired generalized lipodystrophy is characterized by an onset in childhood or adolescence. Patients lose subcutaneous fat from the extremities, the trunk, the neck and face as well as from palms and soles. Concomitant autoimmune disease is common in these patients.

Acquired partial lipodystrophy begins also in childhood and affects primarily the face spreading then down to the neck, shoulders, upper extremities and trunk. There also is an association with autoimmune diseases, especially mesangiocapillary glomerulonephritis that typically develops years after the onset of the lipodystrophy.

All the lipodystrophies share a characteristic phenotype of increased muscular appearance in the areas of fat loss. This may explain why women are more frequently diagnosed with the disease than men, who may not seek medical attention, as their findings may not be as noticeable. Metabolically, the lipodystrophy syndromes are accompanied by severe disturbances of glucose and lipid metabolism with the exception of acquired partial lipodystrophy, which only rarely shows metabolic complications. A hallmark of lipodystrophy is hyperinsulinemia and severe insulin resistance often accompanied by acanthosis nigricans, hirsutism and polycystic ovaries. The defect in glucose homeostasis ranges from abnormal glucose tolerance to overt diabetes mellitus, sometimes requiring insulin doses of over 1000 units per day. The other typical findings are hypertriglyceridemia, chylomicronemia, elevation of free fatty acids and a low level of high-density lipoproteins (HDL). As a consequence, patients may develop xanthomas and are at high risk for acute pancreatitis. Patients can also develop fatty infiltration of the liver, hepatomegaly, abnormal liver function tests and cirrhosis as long-term complications. Serum leptin levels are often low, consistent with the low amount of adipose tissue present in these patients.

The nuclear envelope is the structure that wraps each cell nucleus of higher eukaryotes, and consists of the outer and inner nuclear membranes, the nuclear pore complexes and the nuclear lamina. The nuclear envelope is crucial for proper transmission of genetic information during cell division and for transport processes between cytoplasm and nucleus. The lamina is a meshwork that lines the inner nuclear membrane and is composed of intermediate filament proteins, the nuclear lamins. There are three major lamins, A, B and 
Table 1 Summary of the different lipodystrophy syndromes.

\begin{tabular}{|c|c|c|c|}
\hline Lipodystrophy syndrome & Gene locus/gene/etiology & Onset of fat loss & Fat affected/location of fat loss \\
\hline \multicolumn{4}{|l|}{ Congenital/familial } \\
\hline $\begin{array}{l}\text { Congenital generalized (CGL) } \\
\text { (Berardinelli-Seip syndrome) }\end{array}$ & $9 q 34$ & Birth & All metabolically active fat \\
\hline $\begin{array}{l}\text { Familial partial (FPLD) } \\
\text { (Dunnigan syndrome) }\end{array}$ & $\begin{array}{l}\text { 1q21-22 } \\
\text { Exon } 8 \\
\text { Codon } 482 / 486\end{array}$ & Puberty & Extremities \\
\hline \multicolumn{4}{|l|}{ Acquired } \\
\hline $\begin{array}{l}\text { Acquired generalized } \\
\text { (Lawrence syndrome) }\end{array}$ & $?$ & Childhood & Extremities, trunk, neck, face, palms and soles \\
\hline $\begin{array}{l}\text { Acquired partial } \\
\text { (Barraquer-Simons syndrome) }\end{array}$ & $?$ & Childhood & Face, neck, upper extremities, trunk \\
\hline
\end{tabular}

$\mathrm{C}$, that are divided into two classes. The A-type lamins (A and $\mathrm{C}$ ) arise from alternative splicing of a single gene (LMNA) on chromosome 1q21, whereas the B-type lamins are encoded by separate genes. Lamins $\mathrm{A} / \mathrm{C}$ consist structurally of a globular head, a central rod and a tail domain. They interact with chromatin and proteins of the inner nuclear membrane. The gene encoding one of these inner nuclear membrane proteins, emerin, was found to be mutated in the Xlinked form of Emery-Dreifuss muscular dystrophy (EMD1) years ago. EMD1 and its autosomal counterpart, EMD2, are characterized by progressive muscle wasting and cardiomyopathy with conduction defects. Using positional cloning EMD2 was mapped to human chromosome 1q11-23 and since LMNA mapped to 1q21 and the encoded protein was found in close relationship with emerin in the nuclear envelope, LMNA became a candidate gene. In fact, four mutations in the LMNA gene were identified in patients with EMD2. In parallel, the gene responsible for a familial form of dilated cardiomyopathy (CMD1A) was mapped to chromosome $1 \mathrm{q} 1-21$ and the LMNA gene was suspected due to the clinical similarity of the condition to EMD involving cardiac conduction defects. Five mutations in the LMNA gene could be identified in these families.

Several lines of evidence led to the discovery that $L M N A$ is the gene responsible for FPLD. First, the gene for FPLD was mapped to chromosome 1q21-22. Second, the progressive loss of adipose tissue was reminiscent of the progressive atrophy of skeletal muscle in EMD. And third, the knock-out mouse model for EMD lacking A-type lamins not only exhibited all the symptoms of EMD but also was found to lack white fat. Thus, LMNA became a strong candidate gene for FPLD (10). Cao et al. performed DNA analysis of five Canadian patients with FPLD and their family members, as well as 1000 unaffected healthy subjects (2). All the affected patients were found to be heterozygous for a $\mathrm{G}$ to A point mutation in codon 482 of exon 8 leading to a replacement of arginine (R) by glutamine (Q) (R482Q), whereas none of the 1000 control subjects showed mutations. Shackleton et al. sequenced all 12 exons of LMNA from members of 10 affected families as well as three individuals with FPLD (3). In the affected patients, they found four different mutations in exon 8 of the LMNA gene leading to amino acid substitutions. Three of the mutations, again involved codon 482 and one mutation involved codon 486. No mutations were found in 100 healthy controls. Both groups were able to show co-segregation of the mutations with the disease phenotype. For CGL, Garg et al. performed a genome-wide linkage analysis using short tandem repeats on 17 affected pedigrees and were able to map the locus to chromosome $9 q 34$ (4). This region encodes a variety of genes, but interestingly one of them is the gene for retinoid X receptor $\alpha(\mathrm{RXR} \alpha)$. $\mathrm{RXR} \alpha$ acts as a heterodimer partner for the peroxisome proliferator-activated receptor $\gamma(\operatorname{PPAR} \gamma)$ and is known to be important in adipocyte differentiation. However, a causative role for $\mathrm{RXR} \alpha$ has not yet been demonstrated, and the responsible gene for CGL remains to be identified.

These recent findings represent significant advances in our understanding of the etiology of lipodystrophy. Nonetheless, they raise many new questions. In addition, it becomes readily apparent that, even looking just at the familial forms of lipodystrophy, we are dealing with a very heterogenous group of syndromes, from both a phenotypical and genetic point of view. In fact, the general and the partial lipodystrophies are two genetically distinct diseases involving completely different genes on different chromosomes. On the other hand, different mutations in the same LMNA gene can cause FPLD, EMD1, EMD2 and CMD1A, which involve different tissues. One explanation would be that LMNA mutations affect mesenchymal stem cells, which give rise to both fat and muscle cells (9). The common clinical picture of fat loss in partial and general lipodystrophy implies that either the signaling network of lipid metabolism contains as-yet unidentified key players, such as lamins, or there are several metabolic pathways affecting fat homeostasis. While the hypothesis that $\mathrm{RXR} \alpha$ is involved in CGL agrees with the prevailing concept of adipose metabolism, the recent findings of mutations in LMNA being responsible for 
FPLD are a big surprise. What is the physiological role of lamins? How are they causing these different diseases? How are they interfering with lipid and glucose metabolism? And what is their molecular mechanism of action? There are no immediate answers to these questions, but it is speculated that since mutations in LMNA seem to act through a dominant negative mechanism they could impair dimerization (7). In general, the common feature of progressive atrophy and loss of muscle or fat tissue in the diseases caused by LMNA mutations suggests a role of lamins in cell fate, apoptosis, development and aging. In fact, the expression of lamin $\mathrm{A} / \mathrm{C}$ has been shown to be developmentally regulated (10). Due to their localization at the border between the nucleus and the cytoplasm, it is not clear whether lamins interact with cytosolic or nuclear proteins, but one hypothesis would be that they interact with nuclear transcription factors involved in lipid metabolism. One candidate is sterol regulatory element binding protein-1 (SREBP-1) since mice transgenic for SREBP-1 show a similar picture of lipodystrophy (7). Another candidate may be $\mathrm{RXR} \alpha$, given the linkage findings for CGL.

Lamins also could potentially interfere with glucose and insulin metabolism since other factors besides fat loss could be involved in the metabolic complications of lipodystrophy. On the other hand, could hormonal changes and sex steroids affect the mutated lamins, leading to the onset of the disease during puberty? What is the pathogenesis of the acquired forms of lipodystrophy? They seem to have a very strong immunological component. Could these new findings give us a hint as to what the antigens might be, e.g. lamin $\mathrm{A} / \mathrm{C}$ or related factors or proteins involved in yet a completely different mechanism?

Hopefully, we will have the answers to these questions before another 115 years have passed. This knowledge should provide us with deeper insight into the etiology of the different lipodystrophy syndromes as well as into the physiological and pathophysiological role of the 'mysterious' lamins. In addition, it may help us develop novel diagnostic and therapeutic options for patients with lipodystrophy, EMD, CMD and possibly even obesity and diabetes.

\section{References}

1 Mitchell SW. Singular case of absence of adipose matter in the upper half of the body. American Journal of Medical Sciences 1885 90 105-106.

2 Cao H \& Hegele RA. Nuclear lamin A/C R482Q mutation in Canadian kindreds with Dunnigan-type familial partial lipodystrophy. Human Molecular Genetics 2000 9 109-112.

3 Shackleton S, Lloyd DJ, Jackson SNJ, Evans R, Niermeijer MF, Singh BM, Schmidt H, Brabant G, Kumar S, Durrington PN, Gregory S, O'Rahilly S \& Trembath RC. LMNA, encoding lamin A/ $\mathrm{C}$, is mutated in partial lipodystrophy. Nature Genetics $2000 \mathbf{2 4}$ 153-156.

4 Garg A, Wilson R, Barnes R, Arioglu E, Zaidi Z, Gurakan F et al. A gene for congenital generalized lipodystrophy maps to human chromosome 9q34. Journal of Clinical Endocrinology and Metabolism $1999 \mathbf{8 4} 3390-3394$.

5 Garg A. Lipodystrophies (Review). Americal Journal of Medicine 2000108 143-152.

6 Moir RD, Spann TP, Lopez-Soler RI, Yoon M, Goldman AE, Khuon $S$ et al. The dynamics of the nuclear lamins during the cell cycle-relationship between structure and function (Review). Journal of Structural Biology $2000129324-334$.

7 Hegele RA. The envelope, please: Nuclear lamins and disease. Nature Medicine 20006 136-137.

8 Flier JS. Pushing the envelope on lipodystrophy. Nature Genetics 200024 103-104.

9 Wilson KL. The nuclear envelope, muscular dystrophy and gene expression. Trends in Cell Biology 200010 125-129.

10 Sullivan T, Escalante-Alcalde D, Bhatt H, Anver M, Bhat N, Nagashima K et al. Loss of A-type lamin expression compromises nuclear envelope integrity leading to muscular dystrophy. Journal of Cell Biology 199914 913-919.

Received 11 July 2000

Accepted 20 July 2000 